\title{
An Intelligent approach to enhance the help messages for a compiler - An expert system
}

\author{
Neeta Verma \\ A.P. (CSE DEPT) \\ Inderprastha Engineering College, \\ Ghaziabad, U.P.T.U.Lucknow
}

\author{
Swapna Singh \\ A.P. (MCA DEPT) \\ Inderprastha Engineering College, \\ Ghaziabad, U.P.T.U.Lucknow
}

\begin{abstract}
The traditional approaches used for the development of expert systems are mainly based on the interaction between the knowledge engineer and domain expert .However, the role of user in development process has been undetermined. This paper represents the problem of the users which are not familiar with compiler, at the time of execution of source code. The source code have specific piece of information code written in a programming language $\mathrm{L}$ for which there is an interpreter, compiler, linker and editor .After execution of source code define in any language will provide standard error messages that can not be easily understandable by every users. The actual knowledge based software maintenance system carries out essentially error diagnosis and messages that are not user friendly, so the designed expert system will provide a user friendly help messages for beginners. Domain is specified as error messages of $\mathrm{C}$ compiler.
\end{abstract}

\section{Categories and Subject Descriptors}

This paper is related to concept of Expert System in artificial intelligence.

\section{General Terms}

Design of Expert System.

\section{Keywords}

Expert system, knowledge base, knowledge acquisition.

\section{INTRODUCTION}

An expert system is a software that attempts to reproduce the performance of the human experts, most commonly in a specific problem domain, and is a traditional application and/or sub field of artificial intelligence. Expert system is a branch of artificial intelligence and it is a kind of intelligent computer program, using knowledge base and inference engine to solve the problems solved by experts. Expert system consists of inference engine, knowledge base, knowledge acquiring subsystems, human machine interface and interpreter. knowledgebase contains the knowledge used by human experts, in contrast to knowledge gathered from textbooks or non-experts. A wide variety of methods can be used to simulate the performance of the expert however common to most or all are - first the creation of a so called "knowledgebase" which uses some knowledge The term expert system is reserved for programs representation formalism to capture the subject matter experts (SME) knowledge and second a process of gathering that knowledge from the SME and codifying it according to the formalism, which is called knowledge engineering.

\section{DETECTION OF ERRORS}

1. Syntactic error-due to syntax

2. Logical error -due to a wrong logic

3. Calculation error -due to wrong algorithm.

4. Linker error-due to linking of subroutine.

\section{DEVELOPMENT METHODS EXPERT SYSTEMS Steps to perform \\ - Knowledge formulation and prototyping \\ - Implementation of a complete system \\ - Testing and evaluation \\ - Installation and system integration \\ - Continued maintenance}

The most successful application of Artificial Intelligence (AI) in decision making so far is the development of Decision Support System (DSS), particularly expert system, which is a computer program that act as a 'consultant' or 'advisor' to decision makers. Expert systems are cheaper compared to human experts in the long-term scenario. However, expert systems are relatively costly to develop but easy and cheap to operate. 


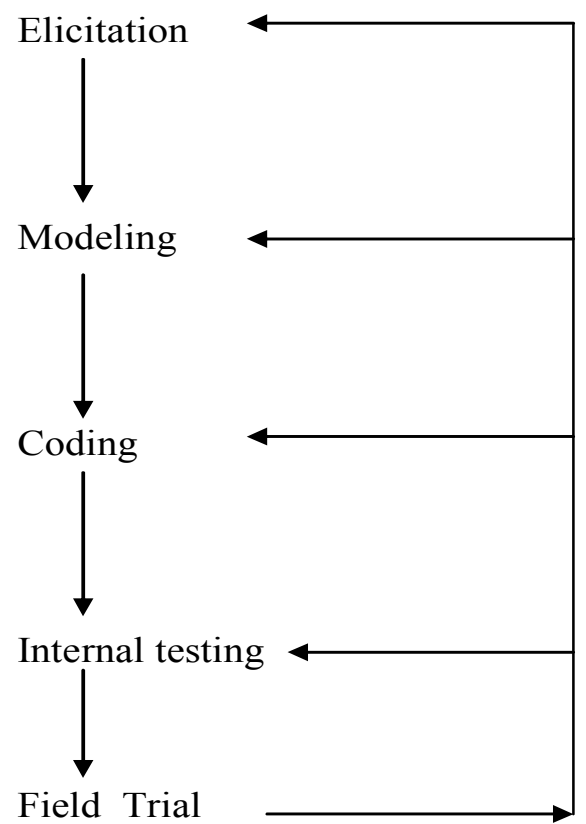

Figure 1. Knowledge-Based System Development

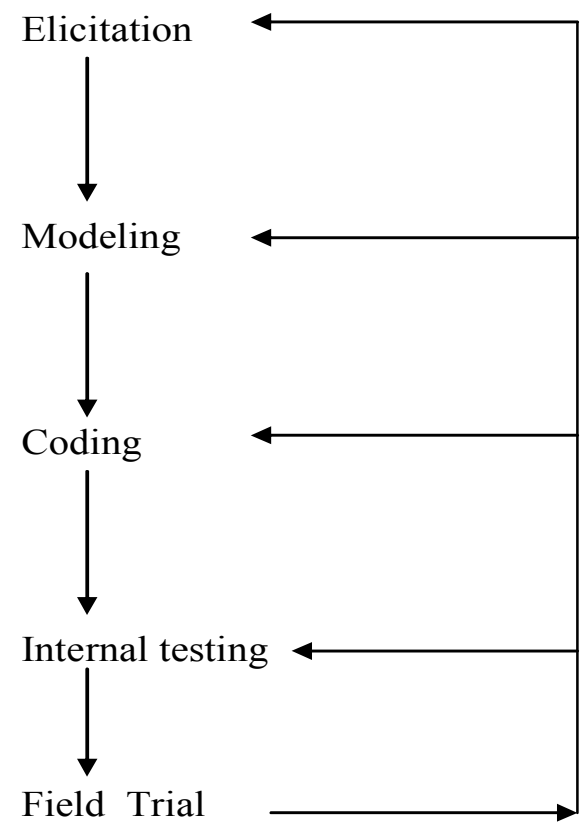

Figure 1. Knowledge-Based System Development

\section{EXPERT SYSTEM DEVELOPMENT}

The power of an expert system is derived from the knowledge of the expert. In the expert system development (fig 1), between the steps of knowledge elicitation and modeling, there is a feedback loop representing the adjustment if inconsistencies due to misunderstanding between domain experts and knowledge engineering. There are also back loop opportunities for other steps for improving the models and codes. The field trial reviews the prototype system and provides domain expert and knowledge engineering with advice to improve the knowledge base.

The fundamental goal of knowledge based approach is to develop an expert program that is capable of solving problems at least as good as human experts.

\subsection{Components of expert system}

A typical expert system consists of four components: knowledge base, user interface, working memory, inference engine.

The knowledge base of an expert system resembles the permanent memory of a human expert. Domain specific knowledge is placed there. The knowledge base is used by inference engine to drive conclusions or solution of problems. The inference engine resembles the thinking part of human cognition process. The inference engine interprets the facts of working memory, applies the knowledge in knowledge base to construct plans and to solve problems. The user interface acts as a pre-processing system that performs syntactic and semantic analysis on user input the relevant information is extracted and stored in working memory.

In the expert system development, our focus is the three main issues, knowledge acquisition, knowledge representation and utilization of knowledge.

\subsubsection{Knowledge base}

Knowledge bases can be represented by production rules. These rules consists of a condition or premise followed by an action or conclusion (IF condition ... THEN action) for my paper the knowledge base will be produced for error messages .For instances (IF prototype error THEN function prototype should be defined), and it will give message how to define function prototype. (IF type mismatch error THEN data type is not the same type ) Production rules permit relationships that makeup the knowledge base to be broken down into manageable units. Having a knowledge base that consists of hundred or thousands of rules can cause a problem with management and organization of the rules. So, the knowledge management is an important issue.

\subsubsection{Knowledge acquisition}

Knowledge acquisition is a process which aims at extracting knowledge, experience and problem solving procedure from one or more domain experts. The knowledge engineering asks the export to report on knowledge that he can directly articulate. The following approaches are

- Interview

- Questionnaires

- Observation of the test performance

- Protocol analysis

The knowledge acquisition phase of expert system development is begin after selecting the problem. In this system the best approach 
for knowledge acquisition is the students who are using the compiler first time.

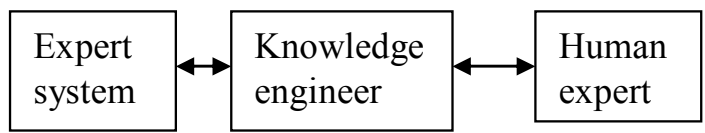

Figure 2. Relationship between Expert System and Human Expert

\subsubsection{Knowledge engineer}

Knowledge engineers have developed a number of principles, methods and tools that have that have considerably improved the process of knowledge acquisition. Knowledge engineers acknowledge that there are different types of experts and expertise; such that methods should be chosen appropriately. The knowledge engineer must make sure that computer has all the knowledge needed to a problem. The knowledge engineering must

choose one or more forms in which to represent the required knowledge as symbols patterns in memory of the computer.

\subsubsection{Inference Engine}

The problem solving model or paradigm organizes and controls the steps taken to solve the problem. One common but powerful paradigm involves chaining of IF-THEN rules to form a line of reasoning. if the chaining starts from a set of conditions and moves towards some conclusion, the method is called forward chaining. if the conclusion is known but the path to that conclusion is not known ,then reasoning backwards is called for, and the method is backward chaining. These problem solving methods are build into program modules called inference engines or inference procedures that manipulate and use knowledge in the knowledge base to form a line of reasoning.

\subsection{KNOWLEDGE REPRESENTATION IN PROLOG}

The knowledge base of system is divided into different parts

- $\quad$ Facts in predicate form, about error types, error localization, the environment and the facts of possible errors.

- $\quad$ Rules, to predicate the error messages according tom the given facts. So the rules can be defined for the matching criteria.

The declarative syntax of prolog programs, in conjunction with their procedural operational semantics, allows for a combination of declarative and procedural representation of knowledge in a cohesive logical framework. Prolog allows only facts and rules to represent the knowledge. Facts in the knowledge base can be made using clauses that do not have a body. e.g.,

Error-is (prototype).

Error-is (Syntactic).

Error-is (Logical ).

Error-is (Calculation).
Error-is (Linker).

The facts of different types of errors messages can be defined. Rules can be defined according to possible errors.

IF Error-is (logical) THEN logic of the statement is not correct\& one example of that logic can be explained.

\section{AN IMPLEMENTATION}

In the existing $\mathrm{C}$ compiler the error messages of the compiled program is not user friendly. For the new user, the error messages are not easily understandable. For exp- error message prototype error, it is not very clear that what is the meaning of prototype error for beginners? The expert system can be designed to make compiler more user friendly. We are planning to design and implement such system for students, those who are beginners. The support can be provide in user friendly way. The knowledge base can be created by the requirement of the students.

\section{ACKNOWLEDGMENTS}

We would like to thank to our Director for providing the full cooperation, support and for helpful comments on an earlier draft of this paper.

\section{REFERENCES}

[1] D. DeGroot and G. Lindstrom , logic Programming: Relations, Functions and Equations. Englewood cliffs,NJ:

Prentice-Hill,1985

[2] Wang Bing, Wang Yuying, Mo Jianjun,"infereence engine designed for real -time monitor and launching decisionmaking expert system" Jor Unal

[3] GuanHuiLing, HanJie, Principle and Practice of Facility Diagnosis Expert System, 1st, China Science Publishing House: BeiJing, 2000.11, pp5-7.

[4] www.epistemics.co.uk/Notes/63-0-0.htm:Knowledge Acquisition

[5] Shu-Hsien Liao (2005). Expert system methodologies and applications -a decade review from 1995 to 2004, Expert Systems with Applications, 28, 93-103.

[6] Joseph Giarratano Gary Riley (2004). Expert Systems: Principles and Programming, Fourth Edition.

[7] Rahman \& Bathnagar, "An Expert System Based Algorithm For Short Term Load Forecast", IEEE Transactions on Power Systems, Vol. 3, No. 2, May 1988.

[8] Markham H.C, “An internet-based expert system for teaching introductory data structures", Proceedings of the seventh annual consortium for computing in colleges central plains conference on The journal of computing in small colleges, pages $155-165,2001$.

[9] Jim Prentzas, et. al., "AWeb-Based ITS Controlled by a Hybrid Expert System”, Proceedings of IEEE International Conference on Advance Learning Techniques (ICALT'01), 2001. 